\section{COOPERATIVISMO, TECNOLOGÍA Y GENERACIONES. LA EXPERIENCIA COOPERATIVA EN ARGENTINA}

\section{COOPERATIVISMO, TECNOLOGIA E GERAÇÕES. A EXPERIÊNCIA COOPERATIVA NA ARGENTINA}

\section{RESUMEN}

A lo largo de este artículo, se exponen proposiciones teóricas a respecto de la Sociedad del Conocimiento, las generaciones y los avances tecnológicos. El objetivo del texto es debatir sobre estas temáticas y exponer una experiencia asociativa que vincula jóvenes y tecnología. Se trata de un grupo de millennials que conforman una cooperativa de trabajo productivo de base tecnológica "La Cooperativa" (LC) ${ }^{1}$ en 2018, centrada en servicios de tecnología 3D que suma el desarrollo de productos propios. Además, a través de la misma, promueven actividades de impacto en un asentamiento territorial. El efecto potencialmente positivo de esta experiencia nos permite identificar el peso del uso de la tecnología en beneficio del mercado de trabajo, en el aporte a disminuir la brecha de desigualdades en oportunidades para una gran proporción de población en Argentina y, el efectivo aprovechamiento de las habilidades de las generaciones de la llamada Era Digital.

Palabras clave: Tecnología. Trabajo. Generaciones. Cooperativismo.

\section{RESUMO}

Ao longo deste artigo são expostas proposições teóricas sobre a Sociedade do Conhecimento, gerações e avanços tecnológicos. $\mathrm{O}$ objetivo do texto é discutir essas questões e apresentar uma experiência associativa que une jovens e tecnologia. É um grupo de millennials que compõe uma cooperativa de trabalho produtivo de base tecnológica "La Cooperativa" (LC) em 2018, focada em serviços de tecnologia 3D que agregam o desenvolvimento de seus próprios produtos. Além disso, por meio dela, promovem atividades de impacto em um assentamento territorial. $\mathrm{O}$ efeito

1 Se utiliza la denominación de la Cooperativa como LC, a modo de mantener el anonimato de los protagonistas de la experiencia conocida y de la cual no se tiene la autorización para divulgar su identidad. 
potencialmente positivo desta experiência nos permite identificar o peso do uso da tecnologia em benefício do mercado de trabalho, na contribuição para reduzir o hiato de desigualdades de oportunidades para uma grande proporção da população argentina e, a efetiva aproveitamento das competências das gerações da chamada Era Digital.

Palavras-chave: Tecnologia. Trabalho. Gerações. Cooperativismo.

\section{INTRODUCCIÓN}

Los principales factores de cambios y sus consecuencias que atraviesan y transforman la sociedad actual se identifican en la globalización, la demografía, el cambio climático y el desarrollo tecnológico (EY, 2016). Estas transformaciones son parte de nuestra cotidianeidad sumida en una constate dinámica de alteraciones que nos confrontan a un panorama incierto de fenómenos hasta hoy poco conocidos.

Estas alteraciones impactan directa e indirectamente en las relaciones sociales así como, también, generan efectos en la economía, la producción, el mercado laboral, las actividades de trabajo, las diversas modalidades asociativas, etc. Y, si bien se trata de procesos de largo aliento, una singularidad de esta época es el ritmo acelerado de los avances tecnológicos con múltiples impactos en el trabajo humano, lo cual resulta un debate que tanto impregna como convoca a las distintas disciplinas académicas. En lo que va del artículo, expondremos materiales sobre tales discusiones, aportando argumentos sobre las generaciones, el uso de la tecnología y una cooperativa de desarrollo 3D en argentina.

En lo que hace al terreno del trabajo humano conviven miradas apocalípticas y esperanzadoras, suele sostenerse de modo que el avance tecnológico acarreará la eliminación y/o el reemplazo de puestos laborales, aunque también que alentará la generación de nuevas oportunidades; cuestiones referidas entre otros por autores y entidades como Castells, Jeremy
Rifkin, Banco Mundial, the Manpower group. No obstante, lo obvio pero preciso priorizar, es que el uso y la implementación de la tecnología dependen de las personas. El hombre "inventa, usa y regula las tecnologías para beneficiar (o dañar) a la humanidad." (BITAR, 2020, p. 25). Aun cuando, el avance exponencial, especialmente de la robótica y de la inteligencia artificial nos confrontan con fuertes desafíos como sociedad que amenazan incluso con alterar este supuesto, de allí lo necesario de abonar ampliando discusiones.

Recordemos aquí que el cooperativismo se basa en los valores de: autoayuda, responsabilidad por los propios actos, democracia, igualdad, equidad y solidaridad. Y, los principios cooperativos son: 1. Adhesión voluntaria y abierta; 2. Control democrático de los miembros; 3. Participación económica de los miembros; 4. Autonomía e independencia. 5. Educación, capacitación e información; 6. Cooperación entre cooperativas; 7 . Interés por la comunidad (ACI, 1996). El objetivo ha sido describir y analizar esta experiencia que vincula jóvenes, tecnología y actividades orientadas a generar un impacto en el desarrollo y mejoramiento de las condiciones de un asentamiento habitacional con poblaciones vulnerables. Para el estudio, se ha trabajado estableciendo contacto y charlas informales con los protagonistas de esta LC desde el 2018 hasta el 2020, complementariamente se han analizado distintas fuentes documentales provistas por los mismos $y$, paralelamente, se han recopilado materiales de prensa sobre la experiencia.

Resta decir que parte de los datos de base del presente análisis derivan de la tesis presentada al Doctorado en Ciencias Empresariales y Sociales, por la candidata Beatriz Elena Plata Martínez en 2020-2021, titulada: "Las tecnologías y su impacto en el trabajo en Argentina, abordaje desde las generaciones como fuerza laboral y la articulación estratégica entre las instituciones educativas, las unidades productivas y los estados", Universidad de Ciencias Empresariales y Sociales (UCES). El objetivo del texto ha sido indagar como las habilidades y capacidades de 
las generaciones de la Era Digital en conjunción con las tecnologías puestas al servicio de las actividades de trabajo en Argentina, tienen impacto positivo. Como parte del trabajo de campo de la investigación, se realizó una muestra no representativa a 167 jóvenes estudiantes secundarios durante el 2019, y se relevó una experiencia argentina (aquí retomada).

De lo anteriormente expresado, este texto se organiza del siguiente modo: un primer y segundo apartado estarán destinados a hilvanar un marco de argumentos sobre la sociedad del conocimiento destacando sus incidencias respecto a los avances tecnológicos y el trabajo. Un tercer apartado estará destinado a retomar el concepto de generaciones y las propuestas de millennial, centennial y alfa, prestando particular atención a su desarrollo en el contexto argentino. En un cuarto apartado, se analizará la experiencia de conformación de $\mathrm{LC}$, dando cuenta de los factores intervinientes, composición, desarrollos elaborados, fines que persigue, etc. y distintas actividades emprendidas. Finalmente, a modo de cierre se sintetizarán las singularidades de la experiencia y se plantearán interrogantes que entendemos de interés en torno a la tecnología y las nuevas generaciones.

\section{SOCIEDAD DEL CONOCIMIENTO Y TECNOLOGÍA}

En el contexto histórico se registran periodos con cambios disruptivos que han marcado y definido en gran parte el rumbo de las sociedades en los modos de producción y por ende en la configuración del trabajo. Han sido cambios y transformaciones profundas, las cuales se demarcan especialmente en cuatro momentos: desde la primera y hasta la actual cuarta revolución industrial. La primera revolución industrial se identifica en el periodo comprendido entre los años 1760 y 1830, la cual marcó el paso de la producción manual (fuerza humana y animal) a la mecanizada. La segunda revolución industrial, desde al de redor de 1850 , con una industria soportada en la electricidad, permitió la manufactura en masa. La tercera revolución industrial desde mediados del siglo XX, llamada la Era de la Informática, con la llegada de la electrónica permitió avances científicos centrales para las Tecnologías de la Información y las Comunicaciones (TIC's). Actualmente, la cuarta revolución industrial ${ }^{2}$ aduce la automatización total de la manufactura; llamada también como la Era de la Digitalización con sistemas ciberfísicos que combinan maquinaria física y tangible con procesos digitales, capaces entre otros, de tomar decisiones. Schwab (2016) plantea que las características del desarrollo tecnológico actual se centran en la velocidad cada vez más exponencial, el alcance con ilimitado acceso a la población y el impacto en los sistemas que "alteran la forma de producir e intercambiar bienes y servicios." (BASCO; CARBALLO, 2017, p. 10).

Hoy con base en los avances de las TICs, la tecnología tiene un papel preponderante, la que Campo (2018) la define como el conjunto de conocimientos (científicos y empíricos) y habilidades o técnicas que sirven para la elaboración de bienes destinados a optimizar la vida del ser humano, el progreso de los pueblos y el medio ambiente. Respecto al uso y aporte de la ciencia frente a estas técnicas durante estos periodos de la historia, Castell (2000) señala que si bien la primera revolución no se basó en la plena exploración de la ciencia para crear técnicas de utilidad para la humanidad, sí se contó con un amplio uso de la información, aplicando y desarrollando el conocimiento ya existente; a posteriori, en la segunda revolución industrial se mostró el papel significativo de la ciencia en fomento de la innovación y con mayor ímpetu durante la tercera revolución, donde la información y el conocimiento se aplican en aparatos con procesamiento de la información/ comunicación y generando conocimiento en un círculo de retroalimentación acumulativo entre la innovación y sus usos.

2 Denominación atribuida al alemán Klaus Schwab, fundador del Foro Económico Mundial como un proyecto de estrategia de alta tecnología del gobierno de Alemania desde 2013 y cuyo objetivo es llevar la producción de este país a una total independencia de la mano de obra humana. 
En otro contexto y respecto a este proceso de desarrollo de la humanidad, Toffler (1980) en su obra La Tercera Ola describe los aspectos que definen la concentración del "Poder" en estos momentos históricos, los cuales clasifica como Sociedades de Tres Olas. Para Toffler, la Sociedad de la Primera Ola se caracterizó por el "Poder" concentrado en la cantidad de tierras que se poseía forjado entre los siglos IX y XV. La Sociedad de la Segunda Ola, desde el siglo XV y hasta el siglo XX, se caracterizó por el "Poder" concentrado en el dominio que se tuviera de la Industria y la cantidad de máquinas que se poseía. La Tercera Ola, desde 1980 en adelante, la denomina como la Sociedad de la Información donde el "Poder" se concentra en el dominio del Conocimiento.

Por su parte, Restrepo (2012), señala que la sociedad de la información hace referencia a la creciente capacidad tecnológica para almacenar cada vez más datos y hacerla circular con mayor rapidez y con mayor capacidad de difusión y, respecto a la Sociedad del Conocimiento alude, que es la apropiación crítica y selectiva de la información protagonizada por todos los ciudadanos que saben qué quieren y cómo aprovechar esta información.

Aún hoy, el humano tiene el control de la tecnología, la mente domina y el "desarrollo informacional es la acción del conocimiento sobre sí mismo como fuente principal de productividad." (CASTELLS, 2000, p. 42). No obstante, esta condición podría variar fundamentalmente en función de los avances en la inteligencia artificial (IA). Siguiendo a Stephen Hawking, la IA es el peligro más inminente para la especie humana dado que el ser humano está limitado biológicamente y en un futuro, le será imposible competir con las máquinas.

La IA se trata básicamente de un desarrollo puesto en máquinas simulando procesos de inteligencia humana. Los avances no están en las máquinas (hardware) sino en el (software) ${ }^{3}$ con-

3 El software engloba todas aquellas actividades las cuales permiten que cualquier máquina con capacidad de procesamiento de información ejecute una función específica; se tienen varios tipos: de base o de ofici- jugando cada vez más capacidad de memoria y velocidad en los procesadores electrónicos.

Otras tecnologías que se destacan con fuerte influencia en la productividad están la computación en la nube, la cadena de bloques, la robótica, bio y nano tecnologías, la biología sintética, la industria 4.0, el agro 3.0, la realidad aumentada, la realidad virtual y la impresión 3D.

De esta forma y en conjunto, la información, el conocimiento, la productividad y el poder, son claves en el accionar social del mundo actual con fuerte influencia del desarrollo tecnológico.

\section{TRABAJO Y TECNOLOGÍA}

Como mencionáramos, las tecnologías aportan procesos, medios y productos de soporte y apoyo a la productividad, la cual asimismo conduce al rápido desarrollo de nuevas capacidades con significativos impactos en los diferentes sectores (social, cultural, económico, político, ambiental, organizacional) y por ende afectando el funcionamiento de las unidades productivas.

Resta claro que la velocidad, el alcance y el impacto de las tecnologías en el mercado de trabajo son intensos. Estos impactos han sido analizados desde 2015 por el Proyecto Milenio. Dicho proyecto con más de 60 nodos en el mundo se aboca al estudio de "Tecnología y Trabajo al 2050", abarca más de 47 países y en el marco de su desarrollo se publicó el informe Work/Technology 2050: Scenarios and actions (2019). Sus resultados sugieren recomendaciones para una transición pacífica a futuro considerando no sólo las consecuencias primarias, sino también las consecuencias secundarias y terciarias del impacto en el trabajo de la inteligencia artificial, la robótica, la biología sintética, la impresión 3D/4D, la bio-impresión, la nanotecnología, la realidad virtual y aumentada, etc. (GUTIÉRREZ, 2016).

na, aplicaciones empresariales (software de gestión a medida y otros), informática para la salud, software de energía y transporte, embebidos en equipos electrónicos, seguridad informática, aplicaciones educativas y videojuegos, entre otros (MINISTERIO DE PRODUCCIÓN Y TRABAJO, 2019). 
Precisamente en el escenario actual y potencial, conviven posiciones optimistas y pesimistas frente al impacto de las tecnologías en la empleabilidad. Las posiciones optimistas sostienen básicamente que cuando la tecnología reemplazó actividades humanas, creó más empleos de los que destruyó. Por el contrario, desde la visión de los llamados tecno-pesimistas las consecuencias de las tecnologías son y serán nefastas, se oponen a su utilización y hasta rechazan la posibilidad de implementar una asignación básica universal como alternativa ante la desocupación, estimando que ello modificaría negativamente la valoración del trabajo para el sostenimiento de la vida humana (GUTIÉRREZ, 2016).

En general, existen múltiples visiones. Por ejemplo, respecto al impacto de las tecnologías y las nuevas tecnologías en el mercado de trabajo Rifkin (1996, p. 25) señala que

Las primeras tecnologías reemplazaban la capacidad física del trabajo humano sustituyendo máquinas por cuerpos y brazos y hoy, las nuevas tecnologías basadas en los ordenadores prometen la sustitución de la propia mente humana, poniendo máquinas pensantes, allí donde existían seres humanos, en cualquiera de los muchos ámbitos existentes en la actividad económica.

Sin embargo, para él las nuevas tecnologías de la información y de las telecomunicaciones tienen la capacidad tanto para liberar como para desestabilizar la civilización del siglo XXI.

Los informes desde organismos como el Foro Económico Mundial (2019) 4 y el Banco Mundial (2019) $)^{5}$ demarcan riesgos globales y

4 Las áreas de preocupación en la encuesta de percepción de riesgos globales en 2018 fueron: 1. vulnerabilidades económicas; 2 . Tensiones geopolíticas; 3 . Tensiones sociales y políticas; 4 . Fragilidades ambientales; 5. Inestabilidades tecnológicas

5 Se hace especial referencia a los riesgos de la no inversión en la educación y la salud de los infantes y su adecuada preparación para los empleos en el futuro, en en los cuales destacan el impacto tecnológico sobre el desempleo y subempleo; advirtiendo que un análisis unidimensional no es suficiente para captar las distintas dimensiones implicadas y sugiriéndose la necesidad de considerar la extensión, la magnitud y la velocidad con que estos cambios se presentan y se presentarán en cada contexto regional y local.

En todo caso, a la hora de hablar de las tecnologías y su impacto en el mercado de trabajo tomando en consideración tanto los argumentos optimistas como los pesimistas, los análisis se orientan hacia la necesidad de adecuación, adaptación y engranaje acorde al modelo de desarrollo en cada territorio. En este sentido, en Argentina "el trabajo en la actualidad, lejos de reducirse a ocupaciones basadas en el conocimiento, o que requieren una gran capacidad de abstracción, mantiene una fuerte heterogeneidad, en la que conviven trabajos, empleos y ocupaciones de muy diferente naturaleza.” (NOVICK, 2010, p. 55). Desde la perspectiva de esta autora, en el mercado de trabajo en Argentina y partiendo de la heterogeneidad de la estructura laboral en el país, argumenta que el desafío es identificar y caracterizar los tipos de trabajo que puedan asociarse con mejores condiciones de empleo y de calidad, con un modelo que integre una estrategia de desarrollo inclusivo con trabajo decente.

\section{GENERACIONES Y TECNOLOGÍA}

Respecto al concepto de "generaciones" y siguiendo a Peirone (2014) su idea la asocia a etapas biológicas de la población humana. Desde el nacimiento hasta el momento de asumir los roles de padres o madres, o con acontecimientos de una época histórica como guerras mundiales, factores culturales (la generación Beat de los años 50 del siglo XX), etc. Actualmente, este enfoque de generación está condicionado al alcance relativo, el uso y la duración

medio de una ola de incertidumbre dado que el ritmo de la innovación se sigue acelerando y la tecnología afecta todos los aspectos de nuestra vida 
de cada innovación tecnológica. El momento bisagra se asocia a cuando las tecnologías análogas dieron paso a las tecnologías digitales impactando en las modalidades de aprendizaje, en el intercambio lingüístico y en las formas de comportamiento socioculturales.

En otro concepto histórico, los estudios de Karl Mannheim (1893-1947) considerado como el fundador del enfoque moderno de las generaciones, abonaron a entender cómo la configuración de una generación no se da solo por el hecho de compartir cercanía en el año de nacimiento, sino por un proceso histórico más amplio que comparten los individuos de igual edad-clase (la generación en sí) (LECCARDI; FEIXA, 2011, p. 17). Es así como se puede afirmar que las generaciones vinculan tiempos de la existencia humana unidos con los cambios sociales, es decir, asociados con la presencia de acontecimientos que rompen con la continuidad histórica y marcan un periodo anterior y un periodo posterior.

Nuestro enfoque refiere a las generaciones poblacionales de las últimas cuatro décadas, las cuales hoy se denominan como de la Era Digital, quienes han crecido al calor de los desarrollos tecnológicos con características particulares de conductas, motivaciones, intereses, anhelos, entre otros, y parte de ellos usan e implementan la tecnología como un eje de vida cotidiana.

En general, los autores no identifican fechas concretas de inicio y fin de cada generación ${ }^{6}$. La llamada generación Y o millennial a quienes señalamos como "jóvenes" está conformada por grupos poblacionales nacidos entre 1980 y los últimos años del cambio de siglo. Siguiendo el informe de Manpowergroup (2018, p. 27) con base en datos de la ONU refiere este término a "aquellas personas nacidas entre 1980 y 1995, que hoy tienen entre 21 y 36 años."

Por otra parte, la llamada generación $\mathrm{Z}$ o centennial está conformada por grupos pobla-

6 Autores como Manpowergroup, consultora Delloite, Accenture, Borrachia, Peirone, entre otros.

7 A hoy están en un rango etario no superior a los 40 años. cionales nacidos desde 1996 y hasta $2010^{8}$. La generación más reciente, llamada "alfa" (primera letra del alfabeto griego) está conformada por grupos poblacionales nacidos desde 2010 en adelante ${ }^{9}$.

Aduciendo a las particularidades propias en el que hacer de estas generaciones con enfoque para nuestro caso en los millennials, se señala que algunos autores sostienen que su principal característica es haber crecido con la influencia directa de las Tecnologías de la información y las comunicaciones TICs y el impacto de la Era de la Digitalización. Centralmente, el uso de los medios de tecnología son parte de su forma de vida, son aficionados a la tecnología del entretenimiento y usuarios asiduos de las redes sociales. Son emprendedores, creadores y privilegian hacer lo que más les gusta. Son apasionados por viajar y compartir estas experiencias a través de las redes sociales. En lo que hace a la educación Díaz-Sarmiento, López-Lambraño, Roncallo-Lafont (2017, p. 199) plantean que esta generación es adepta a oficios y carreras profesionales no tradicionales incluso se forman por medios innovadores como la educación virtual; en general, procuran tener una excelente formación académica.

Respecto al trabajo, Díaz-Sarmiento, López-Lambraño, Roncallo-Lafont (2017, p. 200) describen que los millennials buscan la flexibilidad horaria y la autonomía en el manejo de su tiempo; están interesados en el placer y la diversión en los puestos de trabajo; buscan constantemente nuevas oportunidades; sus expectativas frente al trabajo se centran en la libertad para tomar decisiones, las oportunidades de aprendizaje y desarrollo, la comunicación abierta y el respeto por su estilo de vida; son independientes, delegan responsabilidades, etc.

Estas características de la generación millennial y su interacción en el diario vivir con la tecnología, les ha permitido contar con particulares habilidades y un eje importante en el desempeño como fuerza laboral activa y potencial. Así lo indican informes de diferentes

8 A hoy están en un rango etario no superior a los 24 años 9 A hoy están en un rango etario no superior a los 10 años. 
estudios a nivel global y específicamente para Argentina, entre otras, fuentes referenciales de Manpowergroup, Accenture, Banco Mundial y BID-INTAL.

La Real Academia Española define la "habilidad" como la capacidad/destreza de alguien para desempeñar de manera correcta y con facilidad una tarea o actividad, es decir, una aptitud que puede ser física, mental o social.

En conjunto, las habilidades/destrezas de los millennials se identifican así: a) La rápida adaptación a diferentes entornos: esta generación muestra un constante interés por el movimiento tanto en el entorno de sus actividades personales como laborales, desarrollando una importante capacidad de adaptación. b) La activa interacción social: la propia conectividad permanente con la información circulante, en especial por la web y las redes sociales, los hacen ser abiertos y participativos en los diferentes ambientes sociales. c) La agilidad en la búsqueda de soluciones a situaciones adversas: los estudios indican cómo esta generación surgida de los cambios mundiales de finales del siglo XX en especial, por la internacionalización de la economía y la globalización, navegan por escenarios cambiantes de desafíos y retos, lo que les permite ligereza en dar respuesta a condiciones antagónicas en sus actividades de trabajo (MANPOWERGROUP, 2017; ACCENTURE, 2018).

El informe del Banco Mundial (2019a, p. 50) destaca el desarrollo de habilidades socio conductuales y capacidades características en los grupos generacionales de la era digital, las cuales aún, no pueden ser emuladas totalmente por las máquinas. Se destacan las aptitudes para el trabajo en equipo, la empatía, la capacidad de resolución de conflictos y el manejo de las relaciones.

Ahora bien, expertos como García Canclini (2004) sostienen que la innovación y la adaptación al mercado de trabajo frente a los factores de cambios y sus desafíos actuales y potenciales, son aspectos sustantivos que marcan diferencia a la hora de desarrollar actividades productivas y aunque la generación de los jóvenes millennials y sus subsiguientes, nacen con la impronta de los cambios tecnológicos, también sabemos que el acceso y uso de las herramientas tecnológicas es diferencial.

En este sentido, un aspecto central en la vinculación jóvenes, trabajo y tecnología tiene que ver con el acceso a los recursos más avanzados de informática. En resumidas palabras, como sostiene García Canclini (2004), mientras que la mayoría de los jóvenes de América Latina circulará por la televisión gratuita y las reproducciones piratas, otros navegarán cotidianamente por las opciones más avanzadas de la red. Este manifiesto es una clara alusión a las economías con mejores y peores posibilidades de permitir a la población utilizar la tecnología y que esto redunde en beneficio para el mercado de trabajo. Asimismo, estas dificultades en el acceso a la tecnología implican consecuencias en la formación de diferencias culturales y rangos de diversidad e interculturalidad lejos de cualquier homogeneidad. Entre los jóvenes existen sectores con acceso a las más actualizadas tecnologías y sectores que viven en condiciones variables de pobreza y de limitaciones en el acceso a la educación, al mercado laboral y al consumo de bienes y servicios básicos, con lo cual las desigualdades inter e intra generacionales son una constante y no se aprovechan las propias habilidades tecnológicas de los jóvenes. Por mencionar un ejemplo, Argentina cerró el año 2019 con la cifra de 2/3 de la población total en condiciones de pobreza, datos que en 2020 (durante el primer semestre) se incrementaron a un $40,9 \%$ (ARGENTINA, 2020), es decir, sobre el total de proyección de casi 45,400 millones de habitantes para el 2020, una cifra cercana a 18,560 millones de Argentinos son pobres; y ahondando más en los datos, en el rango etario entre 15 y 29 años de la cifra total estimada de 7 millones de habitantes, el $28,8 \%$ son pobres (ARGENTINA, 2020, p. 6).

Por su parte, respecto a la conectividad de internet en Argentina Basco y Carballo (2017, p. 79) señalan que el país se ubica entre los 3 primeros países de América Latina en penetración de banda ancha fija y de líneas con tecnología 4G, lo cual permite mayor veloci- 
dad y calidad a las conexiones de banda ancha móvil, registrando 15,6 millones de líneas con dispositivos $4 \mathrm{G}$ y penetración del $37 \%$ sobre el total de la población.

El reporte del INDEC (ARGENTINA, 2019) sobre los indicadores al cuarto trimestre de 2018 en accesos y uso de las tecnologías de la información y la comunicación en Argentina, reportaron un $64 \%$ de hogares con acceso a computadora; $80,3 \%$ de hogares con acceso a internet; $42,6 \%$ de población que utiliza computadora $\mathrm{y}$, el $77,7 \%$ de población que utiliza internet.

No obstante, estos datos de cobertura en líneas de banda ancha fija y móvil y de acceso general a la tecnología en la población de Argentina cruzados contra los datos de pobreza, indican una clara desigualdad entre millones de jóvenes quienes no tienen plenas garantías ni reales posibilidades de implementar sus habilidades soportadas en tecnologías.

Cabe destacar por último un dato interesante sobre el desarrollo en Argentina de tecnologías e innovaciones ${ }^{10}$. En la interacción entre tecnologías e innovación, la capacidad, la potencialidad y el emprendimiento local son aspectos destacados por autores argentinos. Más específicamente, el estudio realizado a lo largo de todo el territorio nacional denominado "Argentina Innovadora", Estenssoro y Naishtat (2017, p. 263) identifican múltiples modelos aspiracionales destacados en la transmisión de valores positivos de generación en generación y el arraigo histórico local. Resaltan, además, el escalamiento de los unicornios argentinos a nivel Latinoamericano, tales casos de Despegar, Globant, OLX, Mercado Libre y mencionando diferentes ejemplos de emprendimientos surgidos en momentos difíciles del contexto social, político y económico que no obstante reflejan la alta cualificación del capital social de Argentina.

10 Respecto al concepto de innovación, Joseph A. Schumpeter en su teoría del desarrollo económico (1911), entiende el "desarrollo" como un proceso dinámico de cambios originados desde el propio sistema no afectados por factores exógenos o externalidades, sino sólo endógenos $\mathrm{y}$, plantea cómo la motivación principal del desarrollo microeconómico está centrada en las innovaciones

\section{UNA EXPERIENCIA DE COOPERATIVISMO Y 3D}

Buena parte de los millennials muestran una fuerte capacidad hacia la innovación y frente a lo cual, puede considerarse un ejemplo de ello, la cooperativa "LC". La misma, nuclea jóvenes típicos de esta generación y como describíamos en el apartado anterior, muestran fuerte conexión no solo con la tecnología sino también con la innovación tecnológica.

Más específicamente son un grupo de seis amigos argentinos con residencia en la ciudad de Buenos Aires, de entre 24 y 38 años, la mayoría veinteañeros, con la composición de una mujer y cinco varones. Durante el primer semestre de 2018, deciden consolidar la experiencia de conformar una cooperativa de trabajo como una unidad productiva del sector de servicios.

Con antecedentes comunes de servicio social comunitario, se unieron y planearon la gestión y operación de una idea de base tecnológica. Su objeto inicial se centró en cómo aprovechar sus propias capacidades para obtener un beneficio para sí mismos y para la sociedad. Primeramente, en paralelo a sus actividades individuales se afianzan como equipo de trabajo aportando se propia experiencia y cualificación académica relacionadas con las áreas tecnológicas, unos con formación universitaria de grado en curso y otros titulados, en abogacía, economía, ingeniería de sistemas, ingeniería eléctrica y de programación. Estos factores en conjunto favorecen la creación de LC vinculada a la impresión 3D.

Cabe decir aquí que la evolución de la tecnología hace posible disponer de técnicas para el avance de la ciencia y en especial para su uso e implementación en el mercado de trabajo. Una de las destacadas es la tecnología de impresión 3D definido por Tecnología+Informática (2020), como un proceso de fabricación el cual conlleva a la producción a escala de objetos a partir de una construcción de modelos creados digitalmente. La impresión se logra por la adición de capas de material que son super- 
puestas una sobre otra, de abajo hacia arriba para obtener un objeto final utilizando como material el plástico, siendo los más comunes el ABS y el PLA ${ }^{11}$.

El modelo de negocio de la cooperativa LC se centró en la prestación de servicios en tecnología 3D incluyendo el diseño, la impresión y el escaneo; considerando línea de productos 3D propias. Para lograrlo, se valieron de diferentes recursos. De una parte económicos, con aportes de un subsidio y ahorros personales de los gestores del proyecto para la adquisición inicial de una máquina de impresión 3D (sumado a un proceso de reinversión posterior en la adquisición adicional de más impresoras, materiales y escáner como resultado de las ganancias obtenidas con los productos puestos en el mercado) y, de otra parte técnicos, con procesos de autoaprendizaje y autoconocimiento del uso de esta tecnología desde el punto cero $\mathrm{y}$ hasta el producto terminado apoyados en sus propias habilidades y en tutoriales disponibles en la web.

Como antecedente a la creación de la LC y mencionado anteriormente, los integrantes del equipo desde muy chicos, aún en etapa de estudios secundarios, venían realizando actividades sociales comunitarias, en especial, en contacto con poblaciones limitadas en desarrollo y subsistencia básica de la ciudad de Buenos Aires. Es así como su primera mirada estratégica se concentró en la población del asentamiento ${ }^{12}$ de Saldías. Particularmente este asentamiento se localiza en la Comuna 2 (Recoleta) de la ciudad de Buenos Aires; alrededor de la estación ferroviaria de tren del ramal Belgrano Norte conocido como el Barrio ferroviario Saldías. El censo poblacional de 2010 registró una ocupación de 518 personas (ARGENTINA, 2012). Es una comunidad con servicio de energía eléctrica,

11 PLA y ABS son tipos de filamentos para impresión 3D. El PLA es un termoplástico hecho a base de maíz o caña de azúcar. El filamento ABS es un plástico a base de aceite que es fuerte y resistente.

12 Por asentamiento se entiende un grupo de personas instaladas irregularmente sobre predios estatales o privados, los cuales no pueden ser urbanizados, ni destinados a un uso residencial. no obstante, a la espera de disponer de cloacas, asfalto y gas, entre otros servicios básicos. Durante 2019, avanzaron en la vinculación de habitantes de la población de Villa Oculta, ubicada en la zona del barrio de Villa Lugano y la zona de Villa Crespo, igualmente en la Ciudad Autónoma de Buenos Aires.

Para dar curso a esta experiencia productiva, las funciones del estatuto de creación ${ }^{13}$ contemplan: diseño y Producción de objetos impresos en 3D para el hogar, merchandising y maquetismo; diseño y producción gráfica e imprenta; desarrollo de hardware y software, relacionado a la impresión 3D y a la producción por impresión 3D; brindar servicios de consultoría para soluciones tecnológicas.; realizar el seguimiento técnico y servicio post-vena de hardware y software desarrollados.

En cuanto a los productos desarrollados por la cooperativa tienen una marcada tendencia temática y línea propia, tal como lo registra la nota publicada en Alerta Cultural (2019, online): "entre sus creaciones originales se encuentran objetos para uso diario y decoración como son las masetas, ganchos para las bolsas y, bustos de próceres; lo más vendido son llaveros personalizados que fueron el boom del 2018." Durante el 2019, se desarrollaron entre otros, productos para los fanáticos de Pokémon; trofeos para la liga de futbol femenina; alcancías y muñecos de personajes como Mario Bross, Homero Simpson, etc.

Entre algunas actividades sociales y culturales donde han participado y presentando sus productos están los encuentros en el Parque Patricios de la Ciudad de Buenos Aires como parte de la llamada "Ciudad del Conocimiento" (polo de espacio de impulso a la tecnología, la ciencia, el conocimiento y el desarrollo tecnológico en la ciudad) y encuentros nacionales, como fue el caso de la versión 34 de mujeres realizado en la ciudad de La Plata, Buenos Aires en octubre de 2019.

Tomando en cuenta las últimas actividades señaladas es interesante observar que los fines que persigue la LC, no se limitan solo a la

13 Constituido legalmente en agosto de 2018. 
generación de trabajo para sus propios socios sino antes bien se contempla la promoción de acciones en relación a la comunidad más amplia. De aquí es interesante destacar los vínculos establecidos entre la cooperativa y el entramado del asentamiento. Dado que esta LC, guarda también un sentido social importante, de apoyo hacia los habitantes más vulnerables, la intención de unir su hábitat tecnológico y su capacidad de gestión productiva, el interés está en producir resultados muy significativos para el desarrollo del territorio.

En este sentido, la cooperativa es un ejemplo de experiencia productiva estratégica del sector servicios de cómo el uso e implementación tecnológica conduce a un impacto en la sociedad. Los impactos de esta LC se consolidan tanto en la oportunidad como en la apropiación comunitaria. Por un lado, la oportunidad, a partir de la oferta de espacios de capacitación y la habilitación de espacios laborales (inicialmente indirectos) para miembros de poblaciones desurbanizadas. Asimismo, y muy significativamente la apropiación, con la enseñanza de técnicas del uso de las tecnologías 3D a la población infantil (generación alfa) de estos mismos sectores. De hecho, resultan interesantes las actividades tipo talleres (especialmente los días sábados) que desarrollan con los infantes que interactúan con los instrumentos utilizados en el proceso productivo de esta tecnología $3 \mathrm{D}$, entre otros con cortadores de galletitas y moldes para dibujar, lo cual se realiza directamente en los talleres de la cooperativa.

A poco de su creación y a solo meses de gestión esta $\mathrm{LC}$, ha iniciado un proceso paulatino para generar espacios de empleo y la difusión del conocimiento tecnológico de la impresión 3D. Su foco de atención dirigido al apoyo en comunidades carentes de acceso directo a tecnologías como ésta y el alto déficit en opciones de empleos productivos, dado que son comunidades localizadas en el alto rango de pobreza registrada en Argentina, son ejemplo de cómo aportar desde el uso y la implementación de la tecnología en beneficio para reducir las desigualdades sociales.
Por último, es importante señalar que, en 2019, LC ha tenido un significativo avance respecto al año anterior, logrando generar entre 6 y 10 puestos de trabajo indirectos para pobladores del asentamiento Saldías. Sin embargo, el año 2019 no fue para la cooperativa un periodo ajeno a las incertidumbres y desequilibrios impuestos por la situación macroeconómica del país. La mayor fuente de desajuste y desfasaje está dada por la tasa de cambio del dólar, que afecta directamente su capacidad de adquisición de insumos como el plástico (fundamentalmente provenientes del exterior) y, por ende, la rentabilidad del negocio. Los efectos de tales desequilibrios generan perjuicios dado que el incremento de sus productos impacta en la merma del consumo de los mismos.

Los obstáculos referidos no han generado aun el cierre de la experiencia, sino ocasionado, periodos de cierta discontinuidad o merma en sus actividades. No obstante, los protagonistas de nuestra historia afirman que esperarán condiciones medianamente favorables para reforzar sus actividades siguiendo siempre con su lema y caballito de batalla: "sí pensamos, diseñamos; sí diseñamos, imprimimos, sí imprimimos, transformamos" (LC, 2020).

\section{A MODO DE CIERRE}

A lo largo de este trabajo, hemos hilvanado algunas cuestiones en creciente discusión ligadas al marco actual del acelerado ritmo del desarrollo tecnológico y sus impactos en nuestras sociedades que están afectando la economía, la producción y el mercado de trabajo, así como la estructura misma de las relaciones sociales que en ellas establecemos. En esta misma dirección, la dinámica de nuestras actividades productivas y el trabajo van dando cuenta de transformaciones cada vez más notorias en su fisonomía.

Aquí retomamos una cooperativa de reciente creación en el contexto argentino, más específicamente una experiencia situada en la Ciudad de Buenos Aires, cuyos servicios y producción se basan en la tecnología 3D. El interés de presentar y analizar su estructura, confor- 
mación, fines y actividades que desarrolla, ha tenido la intención de mostrar posibilidades y potencialidades, presentes en el escenario. Suscriben también al interés de prestar atención a las problemáticas existentes y las profundas brechas respecto al uso de la tecnológica, incluso entre aquellos que son parte de una misma generación millennials. En este sentido, la experiencia de esta LC tiene alcance en tanto generadora de un beneficio en las poblaciones menos favorecidas social, económica y culturalmente. Beneficio que se traduce, por una parte, en la generación de alternativas de trabajo productivas; de otra parte, en las actividades de interacción de los infantes en el desarrollo de sus habilidades tecnológicas.

Considerando las cuestiones hasta aquí señaladas, a nuestro entender, apoyar al cooperativismo productivo y de base tecnológica será una tarea sumamente necesaria en el presente y de cara al futuro. Tomando en cuenta que además de la LC aquí esbozada la realidad nos confronta a múltiples experiencias atravesadas por los fines que persigue este ejemplo entendemos que ello nos convoca más que a un cierre a una apertura de debates en torno a los desafíos que conlleva el trabajo, la tecnología, los modos de organización social y laboral. Ante ello destacamos dos de los que consideramos interrogantes de magnitud en futuros desarrollos, por un lado, nos preguntamos, ¿cuán inminente es la discusión sobre el fin social del desarrollo tecnológico?; ¿cuál es el papel de las políticas públicas en este contexto tecnológico?; Y, retomando el concepto de millennials y aún más tomando en cuenta las generaciones futuras ¿qué tipo de acciones y estrategias podrían contribuir a un mayor desarrollo de innovaciones tecnológicas que morigeren las profundas brechas tecnológicas intra e intergeneracionales?.

\section{REFERENCES}

ACCENTURE. La clave está en el aprendizaje, pero no tal como la conocemos: cómo acelerar la adquisición de habilidades en la era de las tecnologías inteligentes. Buenos Aires:
Accenture, 2018.

ACCENTURE. Inclusión en la economía digital. Ponencia presentada en Casos de Innovación Social en organismos y comunidad, Programa de gestión de la innovación, Universidad de Buenos Aires, Ciudad Autónoma de Buenos Aires. Buenos Aires, 2019.

\section{ALERTA CULTURAL. Cooperativa Argenti-} na 3D. 2019. Disponible en: http://www.alertacultural.com/cooperativa-argentina-3d/. Acceso en: 5 fev. 2020.

ARGENTINA. Instituto Nacional de Estadísticas y Censos (INDEC). Incidencia de la pobreza y la indigencia en 31 aglomerados urbanos Primer semestre de 2020. Condiciones de vida, v. 4, n. 13, 2020. Disponible en: https:// www.indec.gob.ar/uploads/informesdeprensa/ eph_pobreza_01_200703093514.pdf. Acceso en: 5 fev. 2020.

ARGENTINA. Instituto Nacional de Censos y Estadísticas INDEC. Mercado de trabajo: tasas e indicadores socioeconómicos (EPH). Informes Técnicos, v. 3, n. 5, 2019. Disponible en: https://www.indec.gob.ar/uploads/informesdeprensa/mercado_trabajo_eph_2trim19ED75D3E4D2.pdf. Acceso en: 5 fev. 2020.

ARGENTINA. Instituto Nacional de Censos y Estadísticas INDEC (2012). Censo nacional de población, hogares y viviendas 2010: censo del Bicentenario: resultados definitivos. Serie B no 2. Buenos Aires: INDEC, 2012.

BASCO, A. I.; CARBALLO, M. Compás Millennial: La generación Y en la era de la integración 4.0. Buenos Aires: BID-INTAL, 2017.

BANCO MUNDIAL. Informe sobre el desarrollo mundial: La naturaleza cambiante del trabajo. Washington: Banco Mundial, 2019a.

BANCO MUNDIAL. Estadísticas de población mundial. 2019. Disponible en: https://datos. 
bancomundial.org/indicador/SP.POP.TOTL. Acceso en: 5 fev. 2020.

BITAR, S. El futuro del trabajo en América Latina: ¿cómo impactará la digitalización y qué hacer?. Washington: Diálogo Interamericano, 2020.

CAMPO, C. La tecnología digital y su impacto en los procesos productivos industriales. Revista Enfoques, Argentina, n. 7, 2018.

CASTELlS, M. La Era de la Información. Economía, sociedad y cultura. La Sociedad Red. 2. ed. Madrid: Alianza Editorial, 2000.

DÍAZ-SARMIENTO, C.; LÓPEZ-LAMBRAÑO, M. Y; RONCALLO-LAFONT, L. Entendiendo las generaciones: una revisión del concepto, clasificación y características distintivas de los baby boomers, X y Millennial. Revista Clío América, v. 11, n. 22, p. 188-204, 2017.

EY. Las ventajas de la disrupción: megatendencias para el 2016 y a futuro. [S.l.]: EY Questions, 2016.

ESTENSSORO, M. E. Y.; NAISHTAT, S. Argentina Innovadora. 2. ed. Ciudad Autónoma de Buenos Aires: Sudamericana, 2017.

FORO ECONÓMICO MUNDIAL WEF. The Global Risks Report 2019. 2019. Disponible en: http://reports.weforum.org/global-risks-2019/chapter-one/. Acceso en: 5 fev. 2020.

GARCÍA CANCLINI, N. Diferentes, desiguales y desconectados. Barcelona: Gedisa, 2004.

GUTIÉRreZ, M. A. Próximas tecnologías: impacto. 2016. Disponible en: https://www. losandes.com.ar/article/proximas-tecnologias-impacto. Acceso en: 5 fev. 2020.
LECCARDI, C.; FEIXA, C. El concepto de generación en las teorías sobre la juventud. Revista Última década, v. 19, n. 34, p. 11-32, 2011.

MANPOWERGROUP. Las carreras de los Millennials: Visión 2020. Datos, cifras y consejos prácticos de los expertos en recursos humanos. [S.l.: s.n.], 2018.

MANPOWERGROUP. Revolución de las habilidades 2.0: los robots no necesitan aplicar:soluciones humanas para la Revolución de las Habilidades. [S.l.: s.n.], 2017.

NOVICK, M. La resignificación del trabajo en la sociedad del conocimiento. Revista Trabajo: El trabajo en la Economía del Conocimiento, México, v. 4, n. 6, p. 51-72, 2010.

OPPENHEIMER, A. Sálvese quien pueda: el futuro del trabajo en la era de la automatización. Ciudad de México: Debate, 2018.

ORGANIZACIÓN INTERNACIONAL DEL TRABAJO OIT. Información Institucional. 2019. Disponible en: www.ilo.org. Acceso en: 5 fev. 2020.

ORGANIZACIÓN PARA LA COOPERACIÓN Y EL DESARROLLO ECONÓMICO -OCDE. PISA: Programa para la Evaluación Internacional de Alumnos. Resultados año 2018. 2019. Disponible en: https://www.oecd. org/pisa/pisaenespaol.htm. Acceso en: 5 fev. 2020 .

PEIRONE, F. El giro copernicano de los jóvenes actuales. Desafíos filosóficos y pedagógicos de una cosmovisión emergente. In: TRABAJO PRESENTADO EN LAS XVII JORNADAS SAPFI 20 ANIVERSARIO: 1994-2014 "Volver a pensar la escuela secundaria y la enseñanza filosófica 20 años después", 17., 2014, Buenos Aires. Anais [...]. Buenos Aires: Colegio Nacional Buenos Aires, 2014. 
PLATA, B. E. (2019). La tecnología en el futuro del trabajo en Argentina: una revisión desde la mirada empresarial, universitaria y del Estado. In: PAZOS, David Villacis (ed.). Una visión internacional de prospectiva multisectorial. Quito: UPACIFICO, 2019. p. 197-227.

RIFKIN, J. EI fin del trabajo. Nuevas tecnologías contra puestos de trabajo: el nacimiento de una nueva era. Buenos Aires: Paidós, 1996.

SCHWAB, K. La cuarta revolución industrial. Debate: World Economic Forum.

Tecnología+Informática (2020). ¿Qué son las impresoras 3D? ¿Para qué sirven?. 2016. Disponible en: https://www.tecnologia-informatica.com/impresoras-3d-que-son-como-funcionan-impresion-3d/. Acceso en: 5 fev. 2020.

TOFFLER, A. La tercera Ola. [S.l.]: Bantam Books, 1980. 